\title{
Validação da Versão Reduzida da Escala de Comportamento Interpessoal para Adolescentes Portugueses
}

\author{
Validation of the Short Version of the Scale for Interpersonal Behavior \\ for Portuguese Adolescents
}

\author{
Paula Vagos", ${ }^{*}$, Anabela Pereira ${ }^{a} \&$ Willem Arrindell ${ }^{b}$ \\ ${ }^{a}$ Universidade de Aveiro, Aveiro, Portugal \\ $\&^{b}$ University of Groningen, Groningen, Province of the Groningen, Netherlands
}

\begin{abstract}
Resumo
Este trabalho apresenta a adaptação linguística para português da Escala de Comportamento Interpessoal - versão reduzida, e a avaliação das suas características psicométricas, em uma amostra de adolescentes tardios (dos 16 aos 21 anos; $N=872$ ). Foi encontrada uma organização interna em quatro dimensões e uma medida geral para os dois componentes avaliados: desconforto ao ser assertivo e frequência de prática de comportamento assertivo. Todas as medidas obtiveram valores aceitáveis de consistência interna e níveis moderados de validade por relação com outras variáveis, face à versão reduzida da Escala de Assertividade de Rathus. Algumas diferenças por sexo foram observadas. Este instrumento mostrou-se útil para ser empregado na avaliação e investigação em psicologia.

Palavras-chave: Validação transcultural, assertividade, análise fatorial confirmatória, Escala de Comportamento Interpessoal - versão reduzida, adolescente.
\end{abstract}

\begin{abstract}
This work presents the Portuguese linguistic adaptation of the short version of the Scale for Interpersonal Behavior and the evaluation of its psychometric characteristics using a sample of late adolescents (aged 16 to 21 years; $N=872$ ). An internal organization of four dimensions was found along with a general measure for the two components under evaluation: discomfort while being assertive and frequency of practicing assertive behavior. All measures obtained acceptable internal consistency values and moderate levels of validity based on the relation to other variables, namely the short version of Rathus Assertiveness Scale. Some sex differences were observed. The instrument showed to be useful to be employed for evaluation and research in psychology.

Keywords: Cross-cultural validation, assertiveness, confirmatory factor analysis, Scale for Interpersonal Behavior - short version, adolescents.
\end{abstract}

As habilidades sociais referem-se ao conjunto de comportamentos praticados em interações sociais que permitem lidar de forma adequada e competente com as exigências dessas situações (Del Prette \& Del Prette, 2010). A assertividade é uma dessas habilidades, que tem sido associada a várias características de personalidade e do funcionamento interpessoal do indivíduo (Arrindell, Sanderman, et al., 1990). Trata-se uma competência social aprendida e circunstancial, ativada unicamente em contextos interpessoais, que consiste na expressão de sentimentos

\footnotetext{
"Endereço para correspondência: Departamento de Educação, Universidade de Aveiro, Campus Universitário de Santiago, Av. Padre Fernão de Oliveira, Aveiro, Portugal 3810-193. E-mail: paulavagos@ua.pt, anabelapereira@ ua.pt e w.a.arrindell@gmail.com

Este trabalho foi parcialmente financiado por uma bolsa da Fundação para a Ciência e Tecnologia, Portugal e pelo Fundo Social Europeu (SFRH / BD / 29574 / 2006). Este trabalho é baseado em resultados utilizados no trabalho de doutorado do primeiro autor.
}

e necessidades pessoais de forma a obter reforço social dos parceiros de interação, quando a obtenção deste reforço está ameaçada (Rich \& Schroeder, 1976). O que define o comportamento assertivo é a sua validade social e cultural, ou seja, determinada resposta será assertiva se for reconhecida pelo grupo como uma autoexpressão e autoafirmação adequada e ao mesmo tempo respeitosa do outro (Rakus, 2006), que permite a obtenção de vantagens moderadas quer para o individuo quer para o seu grupo social, a curto ou médio prazo (Marchezini-Cunha \& Tourinho, 2010).

A assertividade está representada em várias classes de resposta comportamental, como, por exemplo, expressar sentimentos positivos e negativos, tomar a iniciativa, reconhecer e gerir limitações pessoais, ou defender os próprios direitos (Arrindell, Sanderman, Vandermolen, Venderende, \& Mersch, 1988; Arrindell \& van der Ende, 1985). Estas classes de resposta apresentam diferentes níveis de ameaça à obtenção de reforço, seja ele pessoal (por exemplo, ver recusado um convite para sair) ou social 
Vagos, P., Pereira, A. \& Arrindell, W. (2014). Validação da Versão Reduzida da Escala de Comportamento Interpessoal para Adolescentes Portugueses.

(por exemplo, ser excluído do grupo por recusar um pedido realizado por outra pessoa). À semelhança de outras habilidades sociais, a prática da assertividade implica congruência entre o sentir, o pensar e o agir (Azais, Granger, Debray, \& Ducroix, 1999; Del Prette \& Del Prette, 1999), associando-se ao baixo nível de ansiedade (Bandeira, Quaglia, Bachett, Ferreira, \& Souza, 2005), às representações positivas acerca de si próprio e da interação social (Bruch, Kaflowitz, \& Berger, 1988), e ao comportamento verbal e não-verbal concordante (Castanyer, 1996/2005).

Esta competência pode ser de especial utilidade para adolescentes tardios, aqui considerados como indivíduos com idades compreendidas sensivelmente entre os 16 e os 21 anos, que vivem em dependência financeira dos seus progenitores ou cuidadores (Silva, 2004). Nesta fase de desenvolvimento, o adolescente procura fazer a sua transição para o mundo adulto, expressando a sua identidade recentemente descoberta e procurando a integração e aceitação tanto por seus pares como por adultos (Zarret \& Eccles, 2006). As tarefas exigidas para o sucesso social do adolescente comportam a utilização de várias competências sociais, nomeadamente iniciar e manter conversas, tomar a iniciativa de visitar amigos e planejar atividades conjuntas, gerir conflitos e desacordos, partilhar confidências, oferecer apoio emocional e recusar pedidos considerados irrazoáveis (Del Prette \& Del Prette, 1999; Engels, Dekovic, \& Meeus, 2002; Papalaia, Feldman, \& Olds, 2008). A maioria destas competências pode ser considerada assertiva.

Apesar da pertinência teórica e prática do conceito de assertividade, atestada pelas revisões teóricas publicadas na área (Bolsoni-Silva et al., 2006; Heimberg \& Becker, 1981; Murta, 2005), a atenção dada a este conceito em Portugal é ainda escassa, principalmente no que respeita a instrumentos de avaliação. Uma exceção a esta carência é a Escala de Assertividade de Rathus, que foca o comportamento de defesa dos próprios direitos (Detry \& Castro, 1996). Face a esta realidade, torna-se necessário construir ou validar para a língua portuguesa instrumentos de medida que avaliem as várias subclasses de assertividade, em diferentes dimensões do funcionamento psicológico.

A Scale for Interpersonal Behavior avalia, por meio dos seus 50 itens, o desconforto emocional e a prática comportamental assertiva em vários tipos de situações sociais, definindo a assertividade como uma prática comportamental frequente, acompanhada de baixo desconforto em situações interpessoais (Arrindell, Sanderman, et al., 1990). As qualidades psicométricas deste instrumento foram extensamente comprovadas, referindo-se à consistência interna, confiabilidade teste-reteste e validade de constructo, em amostras comunitárias, clínicas e de sujeitos em treino de competências sociais (Arrindell \& van der Ende, 1985; Arrindell et al., 1999; Sarkova et al., 2005). Tal fato tem sustentado a sua adaptação para diferentes países (Arrindell, Bartolini, \& Sanavio, 1990; Arrindell et al., 2001; Bouvard et al., 1999; Eskin, 1993a, 1993b), nomeadamente Portugal. Os resultados da versão portuguesa confirmaram as suas qualidades psicométricas, e indicaram que as mulheres e sujeitos mais velhos apresentam um comportamento assertivo mais frequente, conjugado com níveis mais elevados de desconforto (Vagos \& Pereira, 2010).

Por tratar-se de um instrumento longo, com consequentes limitações à sua utilização, Arrindell, Sanavio e Sica (2002) propuseram uma versão reduzida, selecionando 25 itens que capturassem todos os elementos contidos na escala original. Os resultados obtidos com esta versão atestaram a sua consistência interna e validade de constructo, permitindo de uma forma mais simples e rápida obter resultados e conclusões semelhantes à versão mais longa do instrumento. $\mathrm{O}$ presente trabalho pretende apresentar o processo de tradução e adaptação da versão reduzida da Scale for Interpersonal Behavior para a lingua portuguesa, bem como a análise das suas propriedades psicométricas em adolescentes tardios portugueses.

\section{Método}

\section{Processo de Tradução e Adaptação da Escala} de Comportamento Interpessoal - Versão Reduzida

A versão portuguesa do instrumento foi obtida pelos métodos de tradução e retrotradução (Hambleton, Merenda, \& Spielberger, 2005), efetuados por especialistas alheios ao estudo e fluentes na língua inglesa e portuguesa. Os itens foram primeiro traduzidos da língua inglesa para o português de Portugal por uma professora de português-inglês do ensino secundário e, em seguida, da língua portuguesa para a inglesa por um professor de uma escola de línguas independente. Quatro itens $(5,8,11$ e 16) tiveram de ser revistos uma segunda vez, por incluírem expressões com traduções muito similares em língua portuguesa ("to like" e "to be fond of"). O processo de adaptação linguística dos itens está exemplificado para o item 16 na Tabela 1. A versão original final em língua inglesa e a versão retrotraduzida foram colocadas à consideração do autor original do instrumento, que as considerou equivalentes.

A clareza e compreensão dos itens em língua portuguesa foram avaliadas por reflexão falada, junto a 18 alunos do ensino secundário público em Portugal. Primeiramente, foi-lhes pedido que relatassem dificuldades na compreensão das instruções, itens ou escala com as alternativas de resposta; em seguida, foram questionados sobre o significado que atribuíam a cada item, para garantir que fosse semelhante ao pretendido. Os alunos relataram dificuldades na compreensão das instruções, que levaram à sua simplificação e à alteração da sua apresentação em relação ao formato original da escala. Os itens mostraram-se compreensíveis.

\section{Participantes}

Os participantes deste trabalho foram 872 estudantes do ensino secundário português, sendo 39,4\% homens ( $n$ $=344)$ e $60,2 \%$ mulheres $(n=525)$. Três sujeitos foram omissos nesta informação $(0,3 \%)$. A idade variou entre 16 
Psicologia: Reflexão e Crítica, 27(3), 452-461.

Tabela 1

Exemplo de revisão de item (16) da Escala de Comportamento Interpessoal - Versão Reduzida

\begin{tabular}{ll}
\hline Etapa da tradução & Versão do item \\
\hline Versão original & Saying that you enjoy people telling you they are found of you. \\
Primeira tradução & Dizer que gostas que as pessoas te digam que sentem afeto por ti. \\
Primeira retroversão & To say that you like to be told that people are affectionate about you. \\
Revisão do item & Dizer que gostas que as pessoas te digam que te estimam. \\
Segunda retroversão & Saying that you like it that people tell you they care about you. \\
\hline
\end{tabular}

e 21 anos $(M=16,71 ; D P=1,56)$. Relativamente ao ano de escolaridade, $32,3 \%$ frequentava o $10^{\circ}$ ano $(n=282)$, $35,7 \%$ frequentava o $11^{\circ}$ ano $(n=311)$ e $32 \%$ frequentava o $12^{\circ}$ ano $(n=279)$. Foi verificada uma distribuição semelhante de homens e mulheres por ano de escolaridade $\left(\chi_{(2)}^{2}=0,55 ; p=0,76\right)$. Uma subamostra de 102 estudantes preencheu a versão reduzida da Escala de Assertividade de Rathus (Vagos \& Pereira, 2008). Destes, 43,1\% eram homens $(n=44)$ e $55,9 \%$ eram mulheres $(n=57)$. Um sujeito foi omisso nesta informação (1\%). A média de idade desta subamostra foi de 16,67 anos $(D P=1,33)$, sendo semelhante à da amostra total do estudo $\left(t_{(99)}=-0,3\right.$; $p=0,77)$. Relativamente ao ano de escolaridade, $36,3 \%$ frequentava o $10^{\circ}$ ano $(n=37), 35,3 \%$ frequentava o $11^{\circ}$ ano $(n=36)$ e $27,5 \%$ frequentava o $12^{\circ}$ ano $(n=28)$.

\section{Instrumentos}

Escala de Comportamento Interpessoal-Versão reduzida (ECI-r). Resultou da tradução e adaptação para português da versão inglesa dos 25 itens que compõem a versão reduzida da Scale for Interpersonal Behaviour (Arrindell et al., 2002; Arrindell, Sanderman, et al., 1990). Os itens são avaliados em duas escalas separadas de resposta: uma para intensidade de desconforto ou ansiedade sentida em eventos sociais potencialmente ativadores de uma resposta assertiva (ECI-r desconforto), variando desde 1 (nada) até 5 (extremamente); e outra para a frequência de prática de comportamento assertivo nesses mesmos eventos (ECI-r comportamento), variando desde 1 (nunca) a 5 (sempre).

No trabalho de apresentação da versão reduzida da escala (Arrindell et al., 2002), foram obtidas as mesmas subescalas da sua versão mais longa, compostas pelos mesmos itens em cada uma das escalas de resposta (i.e., ECI-R desconforto e ECI-r frequência de comportamento), e que obtiveram indices adequados de confiabilidade. São elas: (a) Assertividade negativa, que visa a expressão de sentimentos negativos, como pedir a outros mudança do seu comportamento irritativo, expressar discordância, e defender os próprios direitos (por exemplo "Recusar um pedido feito por uma pessoa com autoridade"; $\alpha=0,78$ para desconforto e $\alpha=0,67$ para comportamento); (b) Expressão e gestão de limitações pessoais, que consiste em pedir ajuda face a falhas ou dificuldades, e lidar com crítica e pressão (por exemplo, "Pedir a alguém para explicar uma coisa que não compreendeste"; $\alpha=0,71$ para desconforto e $\alpha=0,67$ para comportamento); (c) Assertividade de iniciativa, que consiste em tomar a iniciativa em situações sociais (por exemplo, "Iniciar uma conversa com um estranho"; $\alpha=$ 0,76 para desconforto e $\alpha=0,72$ para comportamento); e (d) Assertividade positiva, que respeita à expressão de sentimentos positivos, como afeto ou agradecimento (por exemplo, "Reconhecer um elogio acerca da tua aparência pessoal"; $\alpha=0,78$ para desconforto e $\alpha=0,75$ para comportamento). As medidas de escala completa obtiveram também índices adequados de confiabilidade: $\alpha=0,90$ para desconforto e $\alpha=0,85$ para comportamento.

Escala de Assertividade de Rathus - Versão reduzida (RAS-r). Avalia o quanto o comportamento descrito em 11 itens é habitual no repertório do individuo (Arrindell, Sanderman, et al., 1990). A análise das qualidades psicométricas desta versão reduzida demonstrou confiabilidade adequada $(\alpha=0,84)$ e validade de constructo, já que avalia comportamentos referentes à defesa dos próprios direitos, valor e integridade, e à expressão dos próprios sentimentos (Vagos \& Pereira, 2008). Avaliando estes conteudos, a versão reduzida da escala permite claramente diferenciar o constructo de assertividade do constructo de agressividade, o que havia sido problemático com a versão completa desta escala (Arrindell, Sanderman, et al., 1990). Relativamente à sua utilização com a nossa amostra de adolescentes tardios, o índice de consistência interna foi considerado adequado $(\alpha=0,74)$.

\section{Procedimento de Coleta de Dados}

As escolas foram selecionadas no distrito de Aveiro em Portugal, pela sua classificação no ranking nacional de escolas, ou seja, a média de pontuações obtidas por seus alunos em provas finais. Foram selecionadas duas escolas colocadas na média deste ranking, duas colocadas abaixo e duas colocadas acima. A aplicação dos questionários decorreu após ter sido obtida autorização da Direção Geral de Inovação e Desenvolvimento Curricular, que avalia e regula se o estudo segue padrões éticos e de proteção de participantes recolhidos em contexto escolar. Poste- 
Vagos, P., Pereira, A. \& Arrindell, W. (2014). Validação da Versão Reduzida da Escala de Comportamento Interpessoal para Adolescentes Portugueses.

riormente, foi pedida e obtida autorização dos conselhos executivos de cada escola. Cada escola foi responsável pela obtenção do consentimento parental para a participação dos alunos no estudo. Um dos investigadores deslocou-se a cada escola e sala de aula para explicar os objetivos do estudo, e garantir a confidencialidade e anonimato dos dados aos alunos. Os alunos que haviam obtido autorização parental foram então convidados a participar individual e voluntariamente. Nenhum aluno recusou participar. Os questionários foram preenchidos no contexto de sala de aula, demorando cerca de 15 minutos.

\section{Tratamento de Dados}

O tratamento de dados foi feito com recurso ao Statistical Package for the Social Sciences (SPSS; v15.0) e ao Linear Structural Relations (LISREL; v8.0). O primeiro foi utilizado para análise de confiabilidade através do alfa de Cronbach, correlações entre escalas e subescalas pelo recurso ao teste de correlação de Pearson, e diferenças médias obtidas por diferentes grupos sociodemográficos, através do teste de comparação de médias t-student. $\mathrm{O}$ segundo software foi usado para determinar a validade fatorial do instrumento e conteúdos associados ao constructo, enquanto transferidos para uma segunda cultura. Os casos omissos foram tratados por método listwise, ou seja, foram excluídos da análise os sujeitos que tivessem dados faltosos nas suas respostas.

\section{Resultados}

\section{Confiabilidade e Validade de Constructo}

Para obter evidência das qualidades psicométricas básicas do instrumento, que fundamentam a proposta de manutenção da sua organização em quatro subescalas para duas escalas de medida (Arrindell et al., 2002) na população adolescente portuguesa, foi avaliada a confiabilidade e validade de constructo das medidas propostas. Todas obtiveram índices de consistência adequados (valor mí- nimo de 0,70 segundo Nunnaly, 1978) e comparáveis aos obtidos com adultos usando a versão italiana do mesmo instrumento: $\alpha=0,80$ para desconforto e $\alpha=0,68$ para comportamento de assertividade negativa; $\alpha=0,82$ para desconforto e $\alpha=0,77$ para comportamento de expressão e gestão de limitações pessoais; $\alpha=0,76$ para desconforto e $\alpha=0,70$ para comportamento de assertividade de iniciativa; $\alpha=0,81$ para desconforto e $\alpha=0,75$ para comportamento de assertividade positiva; e $\alpha=0,94$ e $\alpha=0,90$ para as medidas completas de desconforto e comportamento, respetivamente.

Para avaliar a validade baseada na relação com outras variáveis, era esperado obter correlações positivas entre os resultados da ECI-r comportamento e os resultados da RAS-r, e negativas entre os resultados da ECI-r desconforto e os resultados da RAS-r. Os resultados da RAS-r correlacionaram-se de forma positiva e significativa com a dimensão de frequência de comportamento da ECI-r, indicando estarem sendo avaliados constructos semelhantes. Com a dimensão de desconforto da ECI-r, os valores de correlação foram sempre negativos, mas apenas significativos para assertividade negativa e assertividade de iniciativa, indicando estarem sendo avaliados constructos não coincidentes (Tabela 2).

\section{Validade Fatorial}

Foram realizadas análises fatoriais exploratórias aos 25 itens que compõem cada uma das escalas, mas os fatores resultantes não foram teoricamente interpretáveis. Estando a estrutura do instrumento validada internacionalmente (Arrindell et al., 2002), a validade fatorial da sua versão portuguesa foi testada por análise fatorial confirmatória, que tem sido procedimento comum nos trabalhos de tradução e adaptação da versão completa escala. Para decidir o método de estimação a utilizar, foi analisada a normalidade dos dados. De acordo com os critérios de Finney e diStefano (2006), foi confirmada a normalidade univariada ( $0 \%$ dos itens da escala de desconforto e $0 \%$ dos

Tabela 2

Correlações entre as Subescalas da ECI-r e RAS-r

(1)

(2)

(3)

(4)

RAS-r ${ }^{\mathrm{a}}$

(1) Assertividade negativa

\begin{tabular}{ccccc}
- & $0,596^{* *}$ & $0,633^{* *}$ & $0,557^{* *}$ & $0,39^{*}$ \\
$0,762^{* *}$ & - & $0,565^{* *}$ & $0,551^{* *}$ & $0,30^{+}$ \\
$0,756^{* *}$ & $0,719^{* *}$ & - & $0,599^{* *}$ & $0,41^{*}$ \\
$0,694^{* *}$ & $0,689^{* *}$ & $0,718^{* *}$ & - & $0,43^{*}$ \\
$-0,26^{+}$ & $-0,16$ & $-0,32^{+}$ & $-0,16$ & - \\
\hline
\end{tabular}

Nota. Correlações para a dimensão de frequência de comportamento são apresentadas acima da diagonal, e as correlações para a dimensão desconforto são apresentadas abaixo da diagonal. ECI-r = Escala de Comportamento Interpessoal - Versão Reduzida; RAS-r $=$ Escala de Assertividade de Rathus - Versão Reduzida.

${ }^{*}$ correlação corrigida em função dos índices de consistência interna das subescalas da ECI-r $\left[\frac{p}{\sqrt{(\alpha E C I * \alpha R A S)}}\right]$
${ }^{*} p<0,001 ;{ }^{*} p<0,01 ;{ }^{+} p<0,05$.
(3) Assertividade de iniciativa

(4) Assertividade positiva

RAS-r ${ }^{\mathrm{a}}$
(2) Expressão e gestão de limitações pessoais$$
p<0,001 ;{ }^{*} p<0,01 ;{ }^{+} p<0,05
$$ 
itens da escala de comportamento com valores de curtose $\geq 7$ e assimetria $\geq 2$ ) e multivariada (curtose multivariada $=1,34$ para a escala de desconforto e 1,16 para a escala de comportamento), pelo que se recorreu ao método de estimação de máxima verossimilhança, sem restrições de parâmetros.

Para cada escala de medida (intensidade de desconforto e frequência de comportamento) foram testados dois modelos: um modelo fatorial de primeira ordem, que divide os 25 itens nas quatro subescalas descritas na constituição do instrumento, e um modelo fatorial de segunda ordem, que organiza os itens nas mesmas quatro subescalas que, por sua vez, são integradas em uma medida geral de escala completa, quer de desconforto quer de frequência de com- portamento. Os resultados do ajustamento destes modelos foram sempre razoáveis, de acordo com as indicações de Diamantopoulos e Siguaw (2000). Apenas os valores associados ao qui-quadrado não foram adequados (Tabela 3 ). $\mathrm{O}$ teste das diferenças do $\chi 2$ entre os modelos de primeira e segunda ordem foi tendencialmente significativo para a medida de desconforto $(\Delta \chi 2=4,95 ; g l=2 ; p<0,10)$; para a medida de comportamento não foi significativo $(\Delta$ $\chi 2=1,62 ; g l=2$ ). Dada a semelhança entre modelos, e para poder realizar comparações internacionais, todas as análises subsequentes tiveram por base os dados relativos ao modelo fatorial de segunda ordem, o qual é consistente com o descrito pelos autores originais da escala (Arrindell et al., 2002).

\section{Tabela 3}

Comparação de Modelos Fatoriais de Primeira e Segunda Ordem, para Escalas de Desconforto e Comportamento da Escala de Comportamento Interpessoal - Versão Reduzida

\begin{tabular}{|c|c|c|c|c|}
\hline & \multicolumn{2}{|c|}{ Desconforto } & \multicolumn{2}{|c|}{ Comportamento } \\
\hline & $\begin{array}{c}\text { Modelo de } \\
\text { primeira ordem }\end{array}$ & $\begin{array}{c}\text { Modelo de } \\
\text { segunda ordem }\end{array}$ & $\begin{array}{c}\text { Modelo de } \\
\text { primeira ordem }\end{array}$ & $\begin{array}{c}\text { Modelo de } \\
\text { segunda ordem }\end{array}$ \\
\hline \multicolumn{5}{|c|}{ Ajustamento comparativo } \\
\hline$\chi^{2}$ & $\begin{array}{c}1129,33 \\
(p<0,001)\end{array}$ & $\begin{array}{c}1134,28 \\
(p<0,001)\end{array}$ & $\begin{array}{c}1329,52 \\
(p<0,001)\end{array}$ & $\begin{array}{c}1331,14 \\
(p<0,001)\end{array}$ \\
\hline Graus de liberdade & 269 & 271 & 269 & 271 \\
\hline NFI & 0,97 & 0,97 & 0,94 & 0,94 \\
\hline NNFI & 0,98 & 0,98 & 0,95 & 0,95 \\
\hline IFI & 0,98 & 0,98 & 0,95 & 0,95 \\
\hline CFI & 0,98 & 0,98 & 0,95 & 0,95 \\
\hline RMSEA & 0,061 & 0,06 & 0,067 & 0,067 \\
\hline \multicolumn{5}{|l|}{ Ajustamento absoluto } \\
\hline MFI & 4,19 & 4,19 & 4,94 & 4,91 \\
\hline \multicolumn{5}{|c|}{ Ajustamento por variância explicada } \\
\hline GFI & 0,91 & 0,91 & 0,89 & 0,89 \\
\hline AGFI & 0,89 & 0,89 & 0,87 & 0,87 \\
\hline \multicolumn{5}{|c|}{ Ajustamento de parcimónia } \\
\hline PGFI & 0,75 & 0,76 & 0,74 & 0,74 \\
\hline AIC & 1241,32 & 1242,27 & 1441,52 & 1439,14 \\
\hline CAIC & 1564,48 & 1553,89 & 1764,68 & 1750,77 \\
\hline \multicolumn{5}{|c|}{ Ajustamento baseado nos resíduos } \\
\hline SRMR & 0,040 & 0,041 & 0,053 & 0,05 \\
\hline
\end{tabular}

Nota. NFI = Normed Fit Index $;$ NNFI = non-normed fit index $;$ IFI = Incremental Fit Index; CFI = comparative fit index $;$ RMSEA = root-mean-square error of approximation; $\mathrm{MFI}=\chi 2$ /graus de liberdade; GFI = goodness-of-fit index; $\mathrm{AGFI}=$ adjusted $\mathrm{GFI} ; \mathrm{PGFI}$ = parsimony $\mathrm{GFI} ; \mathrm{AIC}=$ Akaike Information Criterion $; \mathrm{CAIC}=$ Consistent $\mathrm{AIC} ; \mathrm{SRMR}=$ standardized root mean square residual . 
Vagos, P., Pereira, A. \& Arrindell, W. (2014). Validação da Versão Reduzida da Escala de Comportamento Interpessoal para Adolescentes Portugueses.

Os itens saturaram de forma satisfatória nas subescalas em que foram inseridos $(\lambda>0,35)$, com exceção de 2 itens da medida de frequência de comportamento de assertividade negativa (itens número 6 e 23; Tabela 4). Tendo em consideração os seus valores de saturação, foi realizada uma análise fatorial confirmatória com a exclusão destes itens. Os intervalos de confiança a $90 \%$ para o RMSEA, PDF e ECVI foram sobrepostos para os modelos

Tabela 4 considerando a totalidade dos itens (25 itens) e excluindo estes dois itens ( 23 itens). Em conjunto com os dados de análise de confiabilidade (valor mínimo de 0,60 segundo Diamantopoulos \& Siguaw, 2000), que mostravam que a consistência interna e homogeneidade não melhorava de forma evidente com a exclusão de qualquer um destes itens, foi mantida a estrutura original da escala em 25 itens, assim tornando-a equivalente e estável, por comparação com a sua versão italiana (Arrindell et al., 2002).

Análise Fatorial Confirmatória de Escala de Comportamento Interpessoal - Versão Reduzida

Analise Fatorial Confirmatoria de Escala de Comporta

Desconforto

Frequência de Comportamento

(1)

(2)

(3)

(4)

(1)

(2)

(3)

6. Recusar um pedido de alguém com autoridade

7. Dizer a alguém que achas que te tratou injustamente

14. Pedir a alguém que pare de te aborrecer

18. Recusar produtos ou serviços não satisfatórios

19. Falar com alguém que achas que está a tentar evitar

23. Recusar emprestar uma coisa a um amigo

25. Insistir para que alguém faça a sua parte

3. Pedir a alguém para explicar uma coisa

9. Dizer a alguém que te criticou que tem razão

15. Perguntar a alguém se tu o/a magoaste

20. Pedir desculpa quando cometeste um erro

22. Pedir a alguém que te indique o caminho

24. Admitir que sabes pouco sobre um assunto

1. Iniciar uma conversa com um estranho

2. Contar uma coisa a um grupo de pessoas

12. Participar da conversa de um grupo de pessoas

13. Manter a tua própria opinião face a outros

17. Dar a tua opinião a uma pessoa com autoridade

21. Apresentares-te a alguém

4. Reconhecer um elogio acerca da tua aparência

5. Dizer a alguém que gostas dele/a

8. Dizer a alguém que o/a estimas

10. Reconhecer um elogio sobre algo que fizeste

11. Dizer que gostas que te digam que gostam de ti

16. Dizer que gostas que te digam que te estimam

Saturação no fator de primeira ordem

Confiabilidade composta $\left(\rho_{c}\right)$

$\begin{array}{ll}0,48 & 0,23 \\ 0,67 & 0,64 \\ 0,67 & 0,59 \\ 0,60 & 0,60 \\ 0,70 & 0,62 \\ 0,60 & 0,24 \\ 0,62 & 0,43\end{array}$

$\begin{array}{ll}0,56 & 0,52 \\ 0,59 & 0,58 \\ 0,69 & 0,66 \\ 0,69 & 0,61 \\ 0,68 & 0,64 \\ 0,71 & 0,57\end{array}$

0,46 0,37

0,46 0,43

0,68 0,57

0,68 0,60

0,71 0,67

0,55 0,50
0,49 0,47

0,52 0,67

0,65 0,66

0,66

0,69

0,55

0,76

$\begin{array}{llll}0,99 & 0,96 & 0,99 & 0,86\end{array}$

0,94

0,91

0,64

$0,81 \quad 0,82$

0,76

0,82

$0,68 \quad 0,77 \quad 0,70 \quad 0,76$

Nota. Os itens são apresentados de forma reduzida; AFC - analise fatorial confirmatória, (1) assertividade negativa, (2) expressão e gestão de limitações pessoais, (3) assertividade de iniciativa, (4) assertividade positiva. 
Os índices de confiabilidade composta foram sempre aceitáveis, para as subescalas (Tabela 4 ), e para as medidas completas $\left(\rho_{c}=0,94\right.$ para ECI-r desconforto e $\rho_{c}=0,91$ para ECI-r comportamento). Os índices de saturação dos fatores de segunda ordem sob o fator de primeira ordem foram bastante elevados, conforme pode ser visto na Tabela 4.

\section{Correlações entre Escalas e Subescalas}

Foram sempre observadas correlações significativas e positivas, indicando consistência nos resultados obtidos em função da dimensão avaliada (Tabela 2). Além disso, foram encontradas correlações significativas negativas para as subescalas coincidentes das dimensões de desconforto e frequência de comportamento, a saber: assertividade negativa $(r=-0,139 ; p<0,001)$, expressão e gestão de limitações pessoais $(r=-0,12 ; p<0,001)$, assertividade de iniciativa $(r=-0,266 ; p<0,001)$ e assertividade positiva $(r$ $=-0,199 ; p<0,001)$. Estes dados indicam que quanto maior a intensidade de desconforto sentido, menor a frequência de prática de comportamentos assertivos.

\section{Análise por Variáveis Demográficas}

$\mathrm{Na}$ Tabela 5 são apresentadas as medidas descritivas por gênero para os fatores e medidas gerais das dimensões de desconforto e comportamento da ECI-r.

Tabela 5

Medidas Descritivas de Subescalas da Escala de Comportamento Interpessoal - Versão Reduzida por Gênero

\begin{tabular}{|c|c|c|c|c|c|c|}
\hline & \multicolumn{4}{|c|}{ Gênero masculino } & \multicolumn{2}{|c|}{ Gênero feminino } \\
\hline & Variação & $M$ & $D P$ & Variação & $M$ & $D P$ \\
\hline \multicolumn{7}{|l|}{ ECI-r desconforto } \\
\hline Assertividade negativa & $7-30$ & 14,11 & 5,14 & $7-34$ & 15,24 & 5,48 \\
\hline Expressão e gestão de limitações pessoais & $6-25$ & 10,97 & 4,39 & $6-28$ & 11,3 & 4,47 \\
\hline Assertividade de iniciativa & $6-26$ & 12,24 & 4,15 & $6-28$ & 12,97 & 4,21 \\
\hline Assertividade positiva & $6-26$ & 12,76 & 4,59 & $6-29$ & 13,55 & 5,03 \\
\hline Medida geral & $25-106$ & 50,08 & 16,58 & $25-118$ & 53,05 & 17 \\
\hline \multicolumn{7}{|l|}{ ECI-r frequência de comportamento } \\
\hline Assertividade negativa & $7-35$ & 19,36 & 5 & $7-34$ & 19,35 & 4,63 \\
\hline Expressão e gestão de limitações pessoais & $6-30$ & 19,59 & 4,68 & $6-30$ & 20,27 & 4,49 \\
\hline Assertividade de iniciativa & $6-30$ & 17,88 & 4,06 & $6-29$ & 17,78 & 3,86 \\
\hline Assertividade positiva & $6-30$ & 17,36 & 4,82 & $6-30$ & 17,63 & 4,37 \\
\hline Medida geral & $27-125$ & 74,19 & 16 & $27-142$ & 75,14 & 14,4 \\
\hline
\end{tabular}

Nota . ECI-r = Escala de Comportamento Interpessoal - Versão Reduzida.

Foi verificado que os estudantes do gênero feminino relataram níveis mais elevados de desconforto ao ser assertivo. Esta diferença foi estatisticamente significativa para a medida geral $\left(t_{(867)}=-2,54 ; p=0,011\right)$, e para os fatores de assertividade negativa $\left(t_{(867)}=-2,33 ; p=0,002\right)$, assertividade de iniciativa $\left(t_{(867)}=-2,49 ; p=0,013\right)$ e assertividade positiva $\left(t_{(867)}=-2,33 ; p=0,02\right)$. Na dimensão de frequência de comportamento, a diferença entre os gêneros foi estatisticamente significativa para a expressão e gestão de limitações pessoais $\left(t_{(867)}=-2,27 ; p=0,033\right)$. O gênero feminino reportou maior prática de comportamento assertivo nesta subescala e na subescala de assertividade positiva. Tanto para homens como para mulheres, a frequência de comportamento assertivo foi sempre significativamente ( $p$ $<0,001)$ superior ao desconforto sentido.
A análise por idades produziu correlações significativas positivas, mas muito baixas com a medida completa de frequência de comportamento $(r=0,09 ; p=0,007)$ e com três das suas subescalas: assertividade negativa $(r=0,097$; $p=0,004)$, assertividade de iniciativa $(r=0,068 ; p=0,046)$ e assertividade positiva $(r=0,075 ; p=0,027)$. Estes dados sugerem que quanto maior a idade do indivíduo, maior a frequência de comportamento assertivo praticado nestas subclasses de assertividade.

\section{Discussão}

O presente trabalho propôs-se apresentar o processo de tradução, adaptação e análise das propriedades psicométricas da versão reduzida da Scale for Interpersonal 
Vagos, P., Pereira, A. \& Arrindell, W. (2014). Validação da Versão Reduzida da Escala de Comportamento Interpessoal para Adolescentes Portugueses.

Behavior (Arrindell et al., 2002; Arrindell, Sanderman, et al., 1990). Trata-se de um medida dos componentes emocional e comportamental da assertividade, aplicados a quatro subclasses desta habilidade: expressão de sentimentos negativos e de sentimentos positivos, expressão e gestão de limitações pessoais e tomada de iniciativa. A versão portuguesa do instrumento foi avaliada por reflexão falada e retrotradução, tal como sugerido por Hambleton et al. (2005), o que comprovou a semelhança de significados entre as versões inglesa e portuguesa da escala.

Os resultados de análise psicométrica foram satisfatórios. O modelo de medida proposto para cada componente em avaliação, organizado em quatro subescalas referentes a diferentes subclasses de assertividade que se agrupam em uma medida geral, obteve índices adequados de ajustamento comparativo (por exemplo, NNFI), que são relativamente independentes do tamanho da amostra, ao contrário, por exemplo, do qui-quadrado (Diamantopoulos \& Siguaw, 2000). Os índices de consistência interna foram adequados, embora ligeiramente inferiores aos obtidos com amostras estudantis internacionais (Arrindell et al., 2001; Nota, Arrindell, Soresi, van der Ende \& Sanavio, 2011). A validade de constructo baseada nas relações com outro instrumento de medida de avaliação do comportamento assertivo foi confirmada, concluindo-se que o instrumento avalia duas dimensões homogêneas e pertinentes de assertividade (i.e. desconforto interpessoal e comportamento assertivo).

Os dados encontrados referentes à caracterização demográfica da assertividade considerando conjutamente as suas dimensões afectiva e comportamental, poderá representar uma particularidade da cultura portuguesa, sendo o resultado específico de amostras portuguesas, de jovens adultos e de adolescentes tardios (Vagos \& Pereira, 2010). Em comparação com adolescentes do gênero masculino, as adolescentes portuguesas do gênero feminino reportam prática comportamental mais frequente de expressão e gestão de limitações pessoais, o que já havia sido mostrado em trabalhos europeus (Nota et al., 2011) e norte-americanos (Arrindell et al., 2001; Bridges, Sanderman, Breukers, Ranchor, \& Arrindell, 1991). Trata-se de um tipo de resposta assertiva expectável e socialmente reforçada em mulheres (Carli, 2002), à semelhança da expressão de sentimentos positivos. Embora as mulheres portuguesas pratiquem mais do que os homens esta expressão, a diferença não foi estatisticamente significativa, ao contrário do achado em uma amostra brasileira (Del Prette et al., 2004).

As adolescentes portuguesas reportam, também maior desconforto que os rapazes na expressão de sentimentos positivos e negativos e na tomada de iniciativa, o que já havia sido relatado em trabalhos europeus (Nota et al., 2011; Sarkova et al., 2005). As classes de resposta assertiva de expressão de sentimentos negativos e tentativa de liderança sobre os outros constituem um maior risco social para as raparigas do que para os rapazes (Carli, 2002; Wilson \& Gallois, 1993), o que pode associar-se a elas sentirem-se mais ansiosas do que eles face a este tipo de situações. Ainda, o achado de que os rapazes são menos ansiosos na expressão de sentimentos negativos já havia sido verificado em uma amostra norte-americana (Bridges et al., 1991), sendo também eles quem mais pratica este tipo de comportamentos, de acordo com dados de uma amostra brasileira (Del Prette et al., 2004). Este comportamento é expectável (Wilson \& Gallois, 1993) e socialmente aceite quando praticado por rapazes (Carli, 2002), explicando que eles se sintam menos ansiosos do que as raparigas ao fazê-lo.

$\mathrm{Na}$ presente amostra, tanto rapazes como raparigas apresentaram um nível de comportamento assertivo significativamente superior ao nível de desconforto sentido, o que os coloca na categoria de indivíduos assertive ou non-shy, de acordo com a tipologia de Arrindell, Sanderman, et al. (1990, p. 261). Assim, as raparigas parecem esforçar-se por continuar a praticar os comportamentos que as deixam mais desconfortáveis, e não se verifica o caso de os rapazes deixarem de expressar e gerir as suas limitações pessoais por se sentirem mais desconfortáveis ao fazê-lo. A impossibilidade de comparar as médias obtidas por este grupo com outros grupos de referência adia conclusões relativamente a seus níveis de assertividade, e, consequentemente, à consideração de necessidades de intervenção nesta população.

A idade não se revelou um importante marcador de assertividade, tal como foi encontrado em uma amostra adolescente italiana (Nota et al., 2011), mas contrariamente ao encontrado em uma amostra norte-americana com maior amplitude etária (Arrindell et al., 2001). Os dados deste trabalho indicam uma tendência para o aumento da frequência com que é praticado comportamento assertivo com a idade, o que é de esperar, à medida que o adolescente se vai confrontando e respondendo adequadamente a um maior leque de situações e exigências sociais (Del Prette \& Del Prette, 1999; Silva, 2004).

Algumas limitações devem ser apontadas referentes ao objetivo deste trabalho. $\mathrm{O}$ fato de a amostra ser constituída unicamente por adolescentes tardios limita a generalização das conclusões relativas às qualidades psicométricas do instrumento. Será importante que trabalhos futuros avaliem os resultados deste instrumento com diferentes amostras, clínicas e não clínicas. Outra limitação refere-se à utilização de uma variável única de critério para avaliação da validade de constructo, devendo futuramente ser avaliada a relação entre assertividade e as restantes habilidades sociais, que em conjunto podem servir como fator protetor ao desenvolvimento e bem-estar do individuo (Sarkova et al., 2010).

\section{Conclusão}

Tendo em conta os resultados do processo de tradução, adaptação e análise de suas qualidades psicométricas, a versão portuguesa reduzida da Escala de Comportamento 
Interpessoal poderá ser útil na investigação, diagnóstico e avaliação de competência assertivas e da eficácia de programas de treino de assertividade. A importância do fator idade, que implica maiores oportunidades de vivência social, na promoção da assertividade ficou por concluir no presente trabalho, no que se refere ao grupo restrito de adolescentes tardios.

Este trabalho disponibiliza uma medida de desconforto e comportamento face a várias subclasses de assertividade, algumas dos quais consideradas em outras escalas de habilidades sociais (por exemplo, Inventário de Habilidades Sociais; Del Prette, Del Prette, \& Barreto, 1998). Como para qualquer outra habilidade social, a avaliação da assertividade deve ser multimodal (Del Prette \& Del Prette, 1999), sendo pertinente futuramente a sua avaliação também por recurso a medidas de role-play ou de qualidade do comportamento assertivo praticado, uma vez que mais do que a frequência de comportamento praticado, importa saber se esse comportamento tem validade social e, portanto, é veículo promotor de reforço, integração e bem-estar psicossocial (Castanyer, 1996/2005; Rakus, 2006).

\section{Referências}

Arrindell, W. A., Bartolini, C., \& Sanavio, E. (1990). Versione italiana della "Scale for interpersonal behavior": fedeltà, stabilità e validità concorrente. Psicoterapia Cognitiva e Comportamentale, 5(2), 99-107.

Arrindell, W. A., Bridges, K. R., van der Ende, J., Lawrence, J. S. S., Gray-Shellberg, L., Harnish, R., ...Sanderman, R. (2001). Normative studies with the Scale for Interpersonal Behaviour (SIB): II. US students - A cross-cultural comparison with Dutch data. Behaviour Research and Therapy, 39(12), 14611479. doi:10.1016/S0005-7967(01)00009-2

Arrindell, W. A., Sanavio, E., \& Sica, C. (2002). Introducing a short form version of the Scale for Interpersonal Behaviour (s-SIB) for use in Italy. Psicoterapia Cognitiva e Comportamentale, 8(1), 3-18.

Arrindell, W. A., Sanderman, R., Hageman, W. J. J. M., Pickersgill, M. J., Kwee, M. G. T., Van der Molen, H. T., ... Lingsma, M. M. (1990). Correlates of assertiveness in normal and clinical-samples: A multidimensional approach. Advances in Behaviour Research and Therapy, 12(4), 153-282. doi: 10.1016/0146-6402(90)90004-A

Arrindell, W. A., Sanderman, R., Vandermolen, H., Vanderende, J., \& Mersch, P. P. (1988). The structure of assertiveness - A confirmatory approach. Behaviour Research and Therapy, 26(4), 337-339.

Arrindell, W. A., \& van der Ende, J. (1985). Cross-sample invariance of the structure of self-reported distress and difficulty in assertiveness - Experiences with the Scale for InterpersonalBehavior. Advances in Behaviour Research and Therapy, 7(4), 205-243. doi:10.1016/0146-6402(85)90013-X

Arrindell, W. A., van der Ende, J., Sanderman, R., Oosterhof, L., Stewart, R., \& Lingsma, M. M. (1999). Normative studies with the Scale for Interpersonal Behaviour (SIB): I. Nonpsychiatric social skills trainees. Personality and Individual Differences, 27(3), 417-431. doi:10.1016/S0191-8869(98)00252-9

Azais, F., Granger, B., Debray, Q., \& Ducroix, C. (1999). Cognitive and emotional approach to assertiveness. Encephale, 25(4), 353-357.
Bandeira, M., Quaglia, M. A. C., Bachett, L. S., Ferreira, T. L., \& Souza, G. G. (2005). Comportamento assertivo e sua relação com ansiedade, locus de controle e auto-estima em estudantes universitários. Estudos de Psicologia (Campinas), 22(2), 111121. doi:10.159/SO103-166X2005000200001

Bolsoni-Silva, A. T., Del Prette, Z. A. P., Del Prette, G., Montagner, A. R., Bandeira, M., \& Del Prette, A. (2006). Habilidades sociais no Brasil: Uma análise dos estudos publicados em periódicos. In M. Bandeira, Z. A. P. Del Prette \& A. Del Prette (Eds.), Estudos sobre habilidades sociais e relacionamento interpessoal (pp. 17-45). São Paulo, SPl: Casa do Psicólogo.

Bouvard, M., Arrindell, W. A., Guérin, J., Bouchard, C., Rion, A. C., Ducottet, E., ...Cottraux, J. (1999). Psychometric appraisal of the Scale for Interpersonal Behavior (SIB) in France. Behaviour Research and Therapy, 37(8), 741-762. doi:10.1016/S0005-7967(98)00187-9

Bridges, K. R., Sanderman, R., Breukers, P., Ranchor, A., \& Arrindell, W. A. (1991). Sex differences in assertiveness on the U.S. version of the Scale for Interpersonal Behavior (SIB). Personality and Individual Differences, 12, 1239-1243. doi:10.1016/0191-8869(91)90195-H

Bruch, M. A., Kaflowitz, N. G., \& Berger, P. (1988). Self-schema for assertiveness - Extending the validity of the self-schema construct. Journal of Research in Personality, 2(4), 424-444. doi:10.1016/0092-6566(88)90002-5

Carli, L. (2002). Assertiveness. In J. Worell, Encyclopedia of women and gender: Vol. 1 (pp. 157-167). Gainesville, FL: Academic Press.

Castanyer, O. (2005). Assertividade - Expressão de uma auto-estima saudável (4. ed.). Coimbra, Portugal: Edições Tenacitas. (Traduação de La asertividad: expresion sana autoestima, de O. Castanyer, 1996, Bilbao, Espanha: Desclee de Brouwer)

Del Prette, Z. A. P., \& Del Prette, A. (1999). Psicologia das habilidades sociais - Terapia e educação. Rio de Janeiro, RJ: Vozes.

Del Prette, Z. A. P., \& Del Prette, A. (2010). Social skills and behavior analysis: Historical proximity and new issues. Perspectivas em Análise do Comportamento, 1(2), 4-15.

Del Prette, Z. A. P., Del Prett, A., \& Barreto, M. C. M. (1998). Análise de um Inventário de Habilidades Sociais (IHS) em uma amostra de universitários. Psicologia: Teoria e Pesquisa, 14(3), 219-228.

Del Prette, Z., Del Prette, A., Barreto, M. C. M., Rios-Saldaña, M. R., Ulian, A. L. A. O., Gerk-Carneiro, E., ...Villa, M. B. (2004). Habilidades sociais de estudantes de psicologia: Um estudo multicêntrico. Psicologia: Reflexão e Crítica, 17, 341-350.

Detry, B., \& Castro, M. L. F. (1996). A escala de assertividade de Rathus: Versão portuguesa. In L. Almeida, S. Araújo, M. M. Gonçalvez, C. Machado, \& M. Simões (Eds.), Avaliação psicológica - Formas e contextos (Vol. 4, pp. 357-363). Braga, Portugal: Associação dos Psicólogos Portugueses.

Diamantopoulos, A., \& Siguaw, J. (2000). Introducing LISREL. London: Sage.

Engels, R., Dekovic, M., \& Meeus, W. (2002). Parenting practices, social skills and peer relationships in adolescence. Social Behavior and Personality, 30(1), 3-18. doi:10.2224/ sbp.2002.30.1.3

Eskin, M. (1993a). Reliability of the Turkish version of the Perceived Social Support from Friends and Family Scales, Scale for Interpersonal-Behavior, and Suicide Probability Scale. Journal of Clinical Psychology, 49(4), 515-522. doi:10.1002/1097-4679(199307)49:4<515::AIDJCLP2270490408>3.0.CO;2-K 
Eskin, M. (1993b). Swedish translation of the suicide Probability Scale, Perceived Social Support from Friends and Family Scales, and the Scale for Interpersonal Behavior: A reliability analysis. Scandinavian Journal of Psychology, 34(3), 276281. doi:10.1111/j.1467-9450.1993.tb01122.x

Finney, S. J., \& diStefano, C. (2006). Non-normal and categorical data in structural equation modeling. In G. R. Hancock \& R. O. Mueller (Eds.), Structural equation modeling: A second course (pp. 269-314). Greenwich, CT: Information Age.

Hambleton, R. K., Merenda, P. F., \& Spielberger, C. D. (2005). Adapting educational and psychological tests for cross-cultural assessment. Mahwah, NJ: Lawrence Earlbaum.

Heimberg, R., \& Becker, R. (1981). Cognitive and behavioral models of asserive behavior: Review, analysis and integration. Clinical Psychology Review, 1(3), 353-373. doi:10.1016/0272-7358(81)90011-8

Marchezini-Cunha, V., \& Tourinho, E. Z. (2010). Assertiveness and self-control: A behavioral-analytic interpretation. Psicologia: Teoria e Pesquisa, 26, 295-303. doi:10.159/ SO102-377722010000200011

Murta, S. (2005). Aplicações do treinamento em habilidades sociais: Análise da produção nacional. Psicologia: Reflexão e Crítica, 18, 283-291.

Nota, L., Arrindell, W. A., Soresi, S., van der Ende, J., \& Sanavio, E. (2011). Cross-cultural validity of the scale for interpersonal behavior. International Journal of Adolescent Medicine and Health, 23(1), 39-44. doi:10.1515/ijamh.2011.007

Nunnaly, J. (1978). Psychometric theory. New York: McGrawHill.

Papalaia, D., Feldman, R., \& Olds, S. (2008). Adolescence. In D. Papalaia, R. Felman, \& S. Olds (Eds.), A child's world:

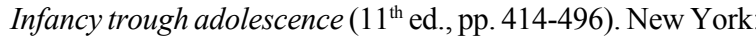
McGraw-Hill Education.

Rakus, R. (2006). Asserting and confronting. In O. Hargie (Ed.), Handbook of communication skills ( $3^{\text {rd }}$ ed., pp. 345-382). New York: Routledge.

Rich, A. R., \& Schroeder, H. E. (1976). Research issues in assertiveness training. Psychological Bulletin, 83, 1081-1096. doi: $10.1037 / \mathrm{h} 0078049$

Sarkova, M., Bacikova-Sleskova M., Orosova, O., Geckova, M., Katreniokova, Z., van den Heuvel, W., \& van Dijk, P. (2010). The associations between assertiveness, psychological well-being and self-esteem in adolescents. In M. Sarkova, Psychological well-being and self-esteem in Slovak adolescents (Unpublished doctoral dissertation). Universidade de Groningen, Netherlands.

Sarkova, M., Katreniakova, Z., Nagyova, I., Geckova, A. M., Orosova, O., van Dijk, J. P., ...van den Heuvel, W. J. A. (2005). The effect of social skills and smoking on the mental health of adolescents. European Journal of Public Health, 15, 170.

Silva, A. (2004). Desenvolvimento de competências sociais. Lisboa, Portugal: Climepsi.

Vagos, P., \& Pereira, A. (2008). A Escala de Assertividade de Rathus: Proposta de uma versão reduzida (RAS-S). In A. P. Noronha, C. Machado, L. Almeida, M. Gonçalves, S. Martins, \& V. Ramalho, Atas da XIII Conferencia internacional de avaliação psicológica: Formas e contextos [CD]. Braga, Portugal: Psiquilibrios

Vagos, P., \& Pereira, A. (2010). Escala de Comportamento Interpessoal: Adaptação para a língua portuguesa. Laboratório de Psicologia, 8(1), 37-49.
Wilson, K., \& Gallois, C. (1993). Assertion and its social context. Oxford, NY: Pergamon Press.

Zarret, N., \& Eccles, J. (2006). The passage to adulthood: Challenges of late adolescence. New Directions for Youth Development, 11, 13-28. doi:10.1002/yd.179 